\title{
Biobancos \\ Una estrategia exigente y esencial para la conservación de muestras biológicas
}

\author{
Biobanks \\ A strict and essentisl strategy conservation of \\ biological samples
}

\author{
Julio César Martínez, Ignacio Briceño, Alejandra Hoyos, \\ Alberto Gómez • Bogotá, D.C. (Colombia)
}

\section{Resumen}

La recolección y conservación de muestras biológicas con fines investigativos es una actividad ligada desde tiempo atrás con la historia y evolución de la ciencia y la medicina; hoy en día, la disponibilidad de bancos de conservación de muestras adecuadamente validadas, está asociada con el desarrollo presente y futuro de la investigación científica.

En general se entiende que un banco de conservación de muestras es un espacio físico que permite mantener en condiciones ideales una serie de muestras biológicas de manera organizada, observando los más altos estándares de calidad, apegados a la normatividad ética y legal establecida para estos casos y cuyo fin es la investigación biomédica tendiente a generar nuevo conocimiento, así como a diversas aplicaciones diagnósticas y terapéuticas.

Los bancos de conservación de muestras constan, más allá de su infraestructura física, de una base de datos que permite conocer en tiempo real y al detalle el estado y condición de cada muestra, además de protocolos y normas claras y perfectamente establecidas para la utilización de las muestras para los proyectos de investigación que las requieran.

La mayoría de los bancos de conservación de muestras nacieron de la necesidad de almacenar muestras biológicas remanentes de alguna investigación biomédica o poblacional, de diferentes campañas de salud, o de muestras recolectadas con propósitos forenses y de criminalística. Posteriormente, con el mejoramiento de las técnicas de conservación de muestras y con el advenimiento de las microtécnicas de análisis molecular, quedó patente la necesidad de institucionalizarlos hasta convertirlos en lo que son hoy, el punto de partida para cualquier proceso investigativo y de generación de nuevo conocimiento, una herramienta científica de primer orden con un potencial de desarrollo virtualmente ilimitado.

Este escrito pretende recrear algunos de los aspectos más importantes del desarrollo de los biobancos, así como resaltar su importancia para la consolidación de una cultura investigativa de calidad, seria y sostenida en el tiempo, que redunde en el desarrollo de la comunidad científica en particular y de la sociedad en general. (Acta Med Colomb 2012; 37: 158-162)

Palabras clave: biobanco, criopreservación, conservación de muestras, especímenes biológicos.
Abstract
The collection and storage of biological samples for research purposes is an activity linked for some time with the history and development of science and medicine. Nowadays, the availability of properly validated sample preservation banks is associated with the current and future development of scientific research.
It is generally understood that a sample conservation bank is a physical space that allows to keep in ideal conditions a number of biological samples in an organized manner, observing the highest standards of quality, according to the ethical and legal norms established for these cases and whose aim is biomedical research, tending to generate new knowledge, as well as diverse diagnostic and therapeutic applications.

Dr. Julio César Martínez: MSc, Investigador Grupo de Genética Humana, Facultad de Medicina, Universidad de La Sabana; Dr. Ignacio Briceño: PhD, Director Grupo de Genética Humana, Universidad de La Sabana; Alejandra Hoyos: Estudiante Facultad de Medicina Universidad de La Sabana, Chía (Cundinamarca); Dr. Alberto Gómez: PhD, Profesor Titular, Instituto de Genética Humana, Facultad de Medicina Pontificia Universidad Javeriana, Bogotá, D.C. (Colombia)

Correspondencia. Dr. Julio César Martínez: MSc,Investigador, Grupo de Genética $\mathrm{Hu}-$ mana. Facultad de Medicina, Universidad de La Sabana, Campus Universitario Puente del Común, km 21 Autopista Norte de Bogotá, Chía, Cundinamarca (Colombia). E-mail: julio.martinez@unisabana.edu.co Recibido:23/IV/2012 Aceptado:20/VIII/2012 
Beyond their physical infrastructure, biobanks consist of a database that allows to know in real time and in detail the status and condition of each sample, as well as protocols and clear and well established guidelines for the use of samples required for research projects.

Most sample conservation banks were born of the need to store remaining biological samples of some biomedical or population research, of different health campaigns, or of samples collected for forensic purposes and criminalistics. Later, with the improvement of sample preservation techniques and the advent of molecular analysis microtechnics, the need to institutionalize them and make them what they are today became evident: the starting point for any research process and of new knowledge generation, a scientific tool of the first order with a virtually unlimited growth potential.

This paper aims to recreate some of the most important aspects of the development of biobanks as well as to highlight its importance for the consolidation of a serious and sustained over time research culture of quality, that benefits the development of the scientific community in particular, and of society in general. . (Acta Med Colomb 2012; 37: 158-162)

Key Words: biobank, cryopreservation, samples conservation, biological specimens.

\section{Introducción}

Desde sus orígenes, el hombre ha dejado constancia de su paso por el universo. Para esto, ha contado con diversos elementos que van desde el testimonio guardado en los restos arcaicos y su entorno, hasta las formas elaboradas por el intelecto como la escritura, a través de la cual se ha perpetuado el conocimiento de la humanidad y registrado su obra.

Paralelo a un proceso evolutivo que podríamos llamar externo o metaorgánico, el hombre ha venido sufriendo otros cambios, también complejos y dinámicos, en el interior de sus células, en la intimidad de sus genes, configurándose y reconfigurándose en un proceso evolutivo interno. De este proceso se conserva testimonio molecular, y desenmarañarlo se ha constituido en un reto para buena parte de la comunidad científica. Con este propósito, el hombre ha desarrollado infinidad de instrumentos y proyectos, el último de ellos es el Proyecto Genoma Humano (1), a través del cual se ha develado lo que se ha denominado en el imaginario popular como "el libro de la vida". Este nuevo mapa molecular es una herramienta que como ninguna otra en la historia de la humanidad está permitiendo, y permitirá aún más en un futuro, resolver muchos interrogantes que pueden llevar a encontrar la clave para comprender el origen y los mecanismos de acción de una buena cantidad de enfermedades, muchas de ellas aun hoy consideradas incurables (2).

Otro aspecto por considerar es la diversidad biológica, en función de la cual algunos países como Colombia tienen el gran privilegio de albergar en su territorio innumerables especies de plantas y animales, algunos de ellos en franco proceso de extinción (3). En el mismo sentido, desde el punto de vista de la especie humana, en el mundo existen un gran número de grupos étnicos, algunos de los cuales han venido experimentando la creciente amenaza de la disolución, bien sea por factores de mestizaje o por otras causas propias de la situación particular de cada familia étnica (4, 5). En un artículo previo, hemos discutido distintos aspectos éticos acerca del trabajo científico en comunidades aisladas, particularmente en el caso específico de las comunidades indígenas de la selva colombiana. Entre los puntos abordados se señalan los siguientes:

a) Los saberes tradicionales frente a la racionalidad científica etnocentrista.

b) La relatividad del "informe de consentimiento" que por norma se solicita a estas comunidades para realizar investigación con ellas.

c) La protección del patrimonio biológico y cultural de estas comunidades ante su posible comercialización.

Finalmente, hemos ya destacado la necesidad de mantener un respeto recíproco y un diálogo permanente sobre las implicaciones de estas investigaciones para unos y otros: investigadores e investigados (5).

En cualquier caso, la extinción de una especie determinada, o la disolución de una familia étnica, acarrea para la humanidad la pérdida de una concreción valiosa e irrecuperable. Por esto, más allá de los programas de conservación y desarrollo sostenible, ha surgido la necesidad de garantizar la preservación del conjunto de características genéticas propias que definen a cada una de estas comunidades, tanto como las que definen a cada especie.

Con esta inquietud como punto de partida se crearon los primeros bancos de conservación de muestras, cuyo fin era proveer los elementos necesarios para exaltar la diversidad biológica y humana, además de garantizar la conservación de especímenes biológicos provenientes de diferentes especies animales o vegetales, así como de los diferentes grupos étnicos. Al tiempo que se buscaba preservarlos, se inicio el estudio del mayor número de moléculas posible en cada caso; es en esta etapa en la que los bancos de conservación de muestras empiezan a constituirse en un instrumento de soporte imprescindible para la investigación científica.

En este propósito, la ciencia y la tecnología contemporáneas han puesto al alcance de los investigadores los instrumentos necesarios para poder analizar una gran cantidad de variables moleculares, partiendo de cantidades mínimas de muestra, lo que garantiza el estudio de la biología humana en el presente y en el futuro. Todo esto asegura que el amplio 
polimorfismo de hoy esté disponible para ser analizado en cualquier momento y por diferentes grupos de investigación alrededor del mundo.

Los primeros biobancos nacieron como iniciativas particulares en respuesta a necesidades individuales que se iban presentando (6); al comienzo sólo eran un lugar para almacenar muestras aisladas. No obstante, con el paso del tiempo y con el potencial científico que representan estos stocks o repositorios, se ha llegado a un nivel de tecnificación importante y hoy son una necesidad en cualquier institución que pretenda establecer un proceso de investigación científico serio a largo plazo, bien sea con fines investigativos, pronósticos o diagnósticos.

En la actualidad, un banco de conservación de muestras biológicas debe proveer, en primer lugar, el espacio físico para guardar de la forma indicada las muestras biológicas; debe estar organizado de manera tal que garantice el soporte técnico necesario para manejo, conservación y transferencia de muestras. También necesita un correcto y dinámico sistema de administración de los especímenes, con el mayor rigor científico y siguiendo los más altos estándares de calidad, todo esto enmarcado dentro de los parámetros éticos establecidos para estos fines (7).

Desde el punto de vista funcional, los bancos de conservación de muestras deben cumplir un doble propósito: primero ser receptores de muestras y garantizar su adecuado almacenamiento y organización; en segundo lugar, oficiar como fuente de muestras para la realización de todo tipo de investigaciones biomédicas relacionadas con el estudio de los mecanismos y alteraciones que afectan al ser humano: investigaciones sobre enfermedades infecciosas, sobre enfermedades de origen genético, neurodegenerativas, sobre diferentes tipos de cáncer, etcétera.

El potencial de investigación que un buen conjunto de muestras puede aportar es inmenso, por lo que no es conveniente que sea desarrollado sólo por la institución administradora de las muestras. Por esta razón, otros grupos de investigación deben poder nutrirse de las existencias de estos bancos, previo establecimiento de los convenios de cooperación pertinentes que garanticen el manejo ético y científico de cada una de las muestras, su óptimo aprovechamiento y, sobre todo, un correcto y juicioso manejo de los resultados obtenidos para que redunde principalmente en beneficio de las comunidades o grupos donantes de las mismas.

\section{Composición de un banco de muestras biológicas}

Existen muchas clases de bancos de muestras, que responden a las necesidades y objetivos de quienes los diseñan pero, en términos generales, un biobanco debe disponer de muestras biológicas representativas de los distintos constituyentes de la función vital como son tejidos, restos tumorales, proteínas, células, plasma, ADN y glóbulos rojos. Las muestras pueden provenir de grupos familiares de pacientes que padecen diversos tipos de enfermedades entre las que se destacan las que tienen componentes predominantemente genéticos o de grupos étnicos que comparten características comunes, o bien de los denominados grupos control, provenientes de la misma población que los grupos de pacientes enfermos.

Las muestras deben ser obtenidas de acuerdo con un objetivo de investigación específico; una vez realizado el análisis primario, éstas se deben almacenar en los congeladores adecuados que permiten garantizar su conservación en condiciones apropiadas para asegurar que cada muestra pueda ser utilizada tiempo después de su obtención. Este sistema de almacenamiento permite contar con un importante potencial de información sobre enfermedades y otros aspectos en la medida que aparezcan nuevos recursos de investigación, nuevas tecnologías, nuevas líneas de investigación, sin que se tenga que convocar a los pacientes para repetidas extracciones.

El almacenamiento masivo de muestras biológicas plantea retos técnicos, logísticos y éticos complejos; desde el punto de vista técnico un primer punto por considerar es el de la aplicación del protocolo de su obtención, que debe asegurar que las muestras están tomadas de forma adecuada y bajo condiciones estándar, lo cual permitirá tener resultados confiables y comparables. La elección de un código de identificación adecuado y suficiente es crucial para la correcta y precisa identificación, clasificación, ubicación y manejo del espécimen.

El siguiente aspecto es el mantenimiento adecuado de la muestra, que se inicia desde la toma y el transporte del sitio hasta el lugar de almacenamiento, lapso en el cual se debe mantener la cadena de frío, que evita que las muestras se deterioren y pierdan sus principales características, haciéndolas inservibles para los posteriores análisis. Una vez realizado el procesamiento y análisis de las muestras, los datos obtenidos deben ser tratados de acuerdo con las normas éticas y legales establecidas para estos casos (7).

La logística debe proveer el espacio físico adecuado y los equipos de mantenimiento indicados según la clase de muestra. Entre los equipos más utilizados están las neveras, congeladores, termos de nitrógeno líquido, espacios a temperatura ambiente y congeladores $-70^{\circ} \mathrm{C}$, entre otros. Además de los protocolos de manejo, debe seguirse rigurosamente la normativa para recolección, procesamiento y transferencia de muestras (8).

Por último, el aspecto ético es de máxima importancia: para atenderlo convenientemente, las instituciones en donde se realiza investigación científica deben contar con los comités éticos que desarrollan funciones como la elaboración y aprobación de los informes de consentimiento para la toma de las muestras, que es el protocolo de autorización explícita y documentada para la utilización de las muestras que se ha denominado "Informe de Consentimiento" o "Consentimiento Informado" (9). La aprobación del componente ético de los proyectos que se desarrollaran a partir del material biológico almacenado debe implicar labores de vigilancia para 
que las normas éticas establecidas sean respetadas durante todo el proceso de toma, almacenamiento, procesamiento, análisis, obtención y comunicación de resultados.

Basándose en diversas opiniones, entre otras la del Grupo Europeo de Ética de la Ciencia y Nuevas Tecnologías de la Comisión Europea, uno de los grupos de discusión que más ha avanzado en la regulación de este tema, el proceso de obtención y procesamiento de material biológico humano para investigación deberá tener presente como mínimo los siguientes principios éticos (10):

- Instalaciones adecuadas, avaladas por autoridades competentes que garanticen el almacenamiento, la conservación y la confidencialidad de la información.

- El profundo respeto hacia el donante y hacia la muestra.

- El respeto a la autonomía del donante. La muestra no deberá ser utilizada sin la autorización del paciente.

- La protección de las personas vulnerables, especialmente aquellas con limitaciones para otorgar consentimiento informado.

- El derecho a la información previa de las condiciones de extracción y el uso potencial que se espera de los tejidos.

- El derecho a evitar la discriminación que podría devenir de la revelación de datos del donante o de su familia a terceros.

- La consideración de estas muestras como potencial fuente de información genética con implicaciones que sobrepasan al propio donante afectando, al resto de su familia y a las generaciones venideras.

- La prohibición de comercialización (obtención de beneficios económicos) a partir de dicha muestra.

\section{Procedimiento de almacenamiento de muestras en un banco biológico}

El procedimiento de almacenamiento de muestras puede presentar ligeras variaciones en cada banco, pero éstos deben compartir criterios. A continuación se presentan algunos de los criterios básicos para este tipo de instituciones:

1. Recolección y almacenamiento unificado de las muestras que van a ser conservadas.

2. Recolección sistemática de datos básicos y clínicos importantes de los donantes.

3. Aplicación del protocolo de almacenamiento específico para cada tipo de muestra.

4. Separación en diferentes alícuotas de cada muestra para poderlas utilizar en diversos procedimientos y para poderlas entregar eventualmente a otros investigadores.

5. Elaboración de formatos para registro y solicitud de muestras para las investigaciones que se realicen a partir de estas muestras.

6. Manejo documentado de resultados correspondientes a cada una de las muestras.

7. Estricta observación de normas de bioseguridad para el manejo de las muestras.

Los más importantes centros de investigación norteamericanos y europeos han demostrado que los bancos de con- servación de muestras adecuadamente dotados y equipados, que garantizan el correcto y seguro almacenamiento de un gran número de muestras biológicas, permiten aumentar la eficiencia de las investigaciones y evitan errores de muestreo (11).

En consecuencia, se logra que las investigaciones tengan mayor validez, a la vez que se permite que otros grupos de investigación tengan acceso a muestras con mayor calidad y confiabilidad debido a que hay mayores garantías en su conservación (8).

El Reino Unido y Suecia son los países que están a la vanguardia en el desarrollo y fortalecimiento de los biobancos, aunque en el mundo existen muchos bancos de conservación de muestras con experiencias exitosas; en el caso de Inglaterra, existe en curso un proyecto de biobanco genético, que intenta obtener muestras de ADN de 500.000 donantes para estudiar la compleja interrelación entre el estilo de vida, los genes y el medio ambiente.

Otro caso interesante es el de la Universidad de Nottingham, donde un grupo de científicos creó el primer banco de ADN del mundo con el objetivo de preservar la información genética de miles de especies en peligro de extinción (12). En el marco de este proyecto, denominado Arca congelada haciendo referencia al Arca de Noé de la tradición judeocristiana, se coleccionaron muestras de ADN y tejidos de las especies en peligro de extinción, para el desarrollo de una reserva en caso de que fracasen los esfuerzos tendientes a evitar su desaparición. Las muestras que serán congeladas a una temperatura de 80 grados centígrados bajo cero, podrían ser utilizadas en el futuro para clonación. Otras experiencias exitosas son CARTaGENE, en Canadá(13), el Swedish National Biobank Program en Suecia (14), y el EuroBioBank del Reino Unido (15). En toda Europa existen numerosos bancos en los que se almacenan ADN, tejidos, muestras de tumores, células madre, etc. En el sector privado hay empresas de biotecnología dedicadas al desarrollo de estos bancos, entre otras están: NeoCodex, Secuvita o Genetrix en el hemisferio norte, y muchas más (6).

En Colombia existen algunos esfuerzos aislados entre los que se pueden destacar, en el ámbito académico, los esfuerzos del Instituto de Investigación de Recursos Biológicos Alexander von Humboldt y, a nivel humano, solamente a manera de ejemplo, el grupo de Neurociencias de la Universidad de Antioquia con un banco para el almacenamiento y estudio de cerebros, tejidos, sangre, plasma y ADN (6), o el Banco Biológico Humano del Instituto de Genética Humana de la Facultad de Medicina de la Pontificia Universidad Javeriana (4).

\section{Regulación de los bancos de conservación de muestras}

Los bancos de conservación de muestras son, en general, altamente heterogéneos, es decir, muy disímiles en cuanto a sus objetivos, diseño, composición y regulación. 
Comparten algunas características comunes, como son la naturaleza gratuita de sus servicios -por cuanto son entidades sin ánimo de lucro-, manteniendo la condición de anonimato de los donantes de las muestras y altos estándares de calidad. También comparten, a nivel global, un aspecto actualmente controvertido como es la ausencia de una legislación clara y seria que los regule, pues en la mayoría de los países que cuentan con este tipo de bancos no existe un marco regulatorio ni una legislación clara que establezca pautas para la tenencia y manejo de muestras biológicas.

En consecuencia, para el futuro de los bancos de muestras, se debe promover el desarrollo de un marco regulatorio y legislativo que abarque los aspectos éticos y legales, así como la armonización de las normas y procedimientos. Una regulación efectiva concertada con otros países para establecer criterios similares para la recolección, almacenamiento y manejo de especímenes resultará en la uniformidad legal que es muy importante para validar los intercambios se realizan entre grupos de investigación de diferentes países en el mundo. Sin embargo, no se debe olvidar que la regulación, tanto legal como ética, es sólo uno de los aspectos que se deben trabajar. Se deberá procurar también la uniformidad de todos los procedimientos, la integración en ensayos clínicos y el trabajo en red (16).

El futuro de los bancos de conservación de muestras biológicas debe enfocarse en desarrollar regulación concertada para todos, en lo posible con un sistema de codificación que responda a criterios internacionales, la creación de un registro nacional y mundial de biobancos, en el que deberán inscribirse todos los organismos que almacenen y gestionen el uso de muestras biológicas con fines de investigación. La existencia de bases de datos abiertas a todos los investigadores a nivel global, es una aspiración a largo plazo que debe empezar a construirse desde ahora. Como ejemplo de ello, el Instituto Roche ha promovido la primera Guía para la utilización de muestras biológicas en la investigación biomédica (17), buscando un manejo uniforme de las muestras, desde el punto de toma de muestra hasta el lugar definitivo de conservación. Estos esfuerzos darán sus frutos a mediano y largo plazo.
En lo inmediato, urge tomar conciencia de la importancia y del potencial que estos bancos representan para el desarrollo de la investigación científica.

\section{Referencias}

1. Venter JC, Adams MD, Myers EW, et al. The sequence of the human genome. Science 2001; 291: 1304-51.

2. Lander, Eric S. Initial impact of the sequencing of the Human genome. Nature 2011; 470: 187-196.

3. Salazar-Holguín Fernando, Benavides-Molineros, Julia, TrespalaciosGonzález, Olga Lucía y Pinzón-Flórez. Informe sobre el Estado de los Recursos Naturales Renovables y del Ambiente, Componente de Biodiversidad Continental 2009. Bogotá: Instituto de Investigación de Recursos Biológicos "Alexander von Humboldt".

4. Gómez, Alberto. El Banco Biológico Humano. Instituto de Genética Humana. Revista Javeriana 1992; 586: 9-11.

5. Gómez, Alberto. Investigación en comunidades aisladas. COLCIENCIAS - Colombia, Ciencia y Tecnología 1998; 16 (1): 8-14.

6. Red temática de investigación cooperativa e informática Regulación de Biobancos. Biomedica-INBIOMED. 2006; 4(17): 1-8.

7. UNESCO - International Bioethics Comitee (16 de Octubre de 2003). Declaración internacional sobre datos genéticos humanos y sobre regulación de biobancos para conservación de muestras.

8. Comisión de criopreservación de muestras biológicas. (2004). Unidad de criopreservación de muestras biológicas Salud pública y Administración Sanitaria. An Sist Sanit Navar 27 (1): 77-86.

9. Veloza L, Wiesner C, Serrano M, Peñaranda N, Huertas A. "Consideraciones éticas y legales de los biobancos para investigación” Revista Colombiana de Bioética, 2010 Vol. 5 No 1[Internet], Disponible en:

10. http://www.bioeticaunbosque.edu.co/publicaciones/Revista/Revista10/ART7.pdf, citado el 23 de febrero de 2012.

11. European Group on Ethics in Science and new Technologies. (21 Julio de 1998). Ethical Aspects of Human Tissue Banking. Opinion of the European Group on ethics in science and new technologies to the european commision.

12. Abascal, Moisés, Teresa y col. Recomendaciones sobre los aspectos éticos de las colecciones de muestras o bancos de materiales humanos con fines de investigación biomédica. Revista Española de Salud Pública 2007; 81(2): 95-111.

13. "The frozen Ark Project" (2010 - 2011) [Internet], disponible en: http://www. frozenark.org, Citado el: 23 de febrero de 2012.

14. "Cart a Gene Project" (2008) [Internet], disponible en: http://www.cartagene. qc.ca, Citado el: 23 de febrero de 2012.

15. "The Swedish National Program" (2003) [Internet], disponible en: http://www. biobanks.se, Citado el: 23 de febrero de 2012.

16. "Euro Bio Bank" (2002-2012) [Internet], disponible en: http://www.eurobiobank org, Citado el: 23 de febrero de 2012

17. Ministerio de la protección social, "Decreto 2493 de 2004" [Internet], disponible en: http://www.ins.gov.co/index.php?idcategoria=6728\#, Citado el: 23 de febrero de 2012.

18. "Guía práctica para la utilización de muestras biológicas en investigación Biomédica", disponible en http://www.institutoroche.es/web/pdf/guia/pdf_completo. pdf, Citado el: 23 de febrero de 2012. 